\title{
Detection of bacterial phosphatase activity by means of an original and simple test
}

\author{
G. SATTA, G. GRAZI, P. E. VARALDO, AND R. FONTANA \\ From the Institute of Microbiology, University of Genoa Medical School, Genoa, Italy
}

SUMMARY A new test for the detection of bacterial phosphatase activity has been devised. The test is performed using agar media containing both methyl green (MG) and phenolphthalein diphosphate (PDP); in these media phosphatase-producing strains grow deep-green-stained colonies whereas non-producing strains do not. A total of 739 different strains were tested, including 593 staphylococci, 95 micrococci, 11 streptococci, 10 corynebacteria, 14 enterobacteria, and 16 candidae. All strains found phosphatase-positive according to the conventional phosphatase test displayed deep-greenstained colonies on MG-PDP media, whereas all phosphatase-negative strains showed unstained colonies on the same media. The main advantages of the present phosphatase test as compared with other conventional ones are that it is more simple to perform, it can reveal the phosphatase activity of colonies grown in deep agar, and can be incorporated into commercial multitest kits.

The production of phosphatase is acknowledged as a key-test for both classification and routine identification of the Micrococcaceae (Baird-Parker, 1963; Schleifer and Kloos, 1975; Subcommittee on the Taxonomy of Staphylococci and Micrococcci, 1976; Oeding and Digranes, 1977). Phosphatase production has also been studied in many other microorganisms, including streptococci (Taketo and Taketo, 1974), corynebacteria (Bray and King, 1943), enterobacteria (Bayliss et al., 1948; Wolf et al., 1972; Bhatti and Done, 1974), anaerobic bacteria (Porschen and Spaulding, 1974), and yeasts (Bayliss et al., 1948; Smith et al., 1973).

In routine clinical microbiology phosphatase activity is generally tested by procedures that require a further step after the inoculation of the medium. Such an operation is relatively time-consuming and can easily lead to either death or contamination of the assayed colonies.

We describe a new phosphatase test requiring only the inoculation of a suitable medium. The test is performed by growing micro-organisms on agar plates containing both phenolphthalein diphosphate and methyl green and results in a deep-green staining of phosphatase-producing colonies. Therefore, phosphatase production of many different bacterial species is detected with a one-step colonystain technique.

Received for publication 3 October 1978

\section{Material and methods}

\section{MICRO-ORGANISMS}

A total of 593 staphylococci, 95 micrococci, 11 streptococci, 10 corynebacteria, 14 enterobacteria, and 16 candidae were studied. Most of these strains were isolated from clinical material examined in the Clinical Bacteriology Laboratory of our Institute. Some strains were also obtained from the skin, nose, and throat of healthy volunteers. Staphylococci were identified according to Kloos and Schleifer (1975) and micrococci according to Kloos et al. (1974). Identification of streptococci, corynebacteria, enterobacteria, and candidae was carried out according to current criteria (Lennette et al., 1974).

\section{TEST MEDIA}

M9 medium (Satta et al., 1977) was used as base medium for the new phosphatase test herein described with all strains studied. Depending on the micro-organisms to be tested, several other media were examined for their potential use as base media and are listed in the Results section. Both methyl green (MG; Carlo Erba) and phenolphthalein diphosphate (PDP; Serva) were added from Seitzfiltered master solutions to the base media (melted and kept at $44^{\circ} \mathrm{C}$ ) to obtain the required final concentrations. Test media were then poured into petri dishes and allowed to solidify. Isolated colonies of strains to be tested were picked up by a loop and stabbed into the test medium. 
EVALUATION OF GREEN COLONY STAIN The degree of green staining of colonies grown on MG-PDP media was scored as follows: - no stain; \pm weak stain;,+++ progressive intensity of staining.

DETERMINATION OF PHOSPHATASE ACTIVITY BY CONVENTIONAL METHODS

Conventional determination of phosphatase activity was performed basically according to King (1943) and Barber and Kuper (1951).

\section{Results}

DEVELOPMENT AND EVALUATION OF MG-PDP PHOSPHATASE TEST

In the course of an investigation of substances able selectively to inhibit some staphylococcal species, we noticed that, when an MG and PDP containing medium was used, some colonies showed a deepgreen staining while others did not. On media containing either MG or PDP alone, no green colony staining was observed. Furthermore, all of the deepgreen colonies grown on MG-PDP medium, but none of the unstained ones, turned red when the plates were exposed to ammonia vapours. These findings suggested the possibility of a relation between deep-green staining and phosphatase activity.

Using M9-base medium, several MG and PDP concentrations were assayed in order to obtain the optimal differential staining of phosphatase-positive and -negative micro-organisms. Optimal concentrations of MG and PDP varied significantly with the different groups of micro-organisms. Moreover, the minimal concentration of MG necessary to produce a deep stain in phosphatase-positive colonies was found to be dependent on PDP concentration and to become progressively lower as the PDP concentration was increased.

With the Micrococcaceae, the lowest concentrations of MG and PDP that allowed for an optimal differential staining of phosphatase-positive and -negative colonies were 25 and $200 \mu \mathrm{g} / \mathrm{ml}$, respectively. Concentrations for streptococci were similar (25 $\mu \mathrm{g} / \mathrm{ml} \mathrm{MG}$ and $400 \mu \mathrm{g} / \mathrm{ml}$ PDP). Higher concentrations of both substances were needed for optimal differential staining of phosphatase-positive and -negative colonies of corynebacteria $(50 \mu \mathrm{g} / \mathrm{ml}$ MG and $800 \mu \mathrm{g} / \mathrm{ml}$ PDP). Enterobacteria needed particularly high concentrations of both MG $(500 \mu \mathrm{g} / \mathrm{ml}$, that is, 10 to 20 times higher than that needed for the other organisms) and PDP (800 $\mu \mathrm{g} / \mathrm{ml})$. With candidae, the phosphatase activity in the normal M9-base medium could not be detected either by the MG-PDP or by the conventional test. When the $\mathrm{pH}$ of the M9-base medium was lowered to $5 \cdot 5$, the phosphatase activity became detectable by the conventional test and only the phosphatasepositive colonies stained deep-green with the MG-PDP test. This indicates that the phosphatase of the candidae tested is an acid phosphatase. Other fungi that bear acid phosphatases have already been described (Bayliss et al., 1948; Smith et al., 1973).

The differences in the MG concentration necessary to perform the MG-PDP phosphatase test in various groups of micro-organisms are likely to be dependent upon the varying permeability of different microorganisms to this dye. In particular, the very high MG concentration necessary to detect the phosphatase activity of enterobacteria can easily be explained by the fact that Gram-negative strains are less permeable to many substances, including dyes, as compared to Gram-positive strains. The differences in the PDP concentration may depend on quantitative differences in the phosphatase activity expressed. The strains requiring higher PDP concentrations usually gave weaker positive phosphatase reactions by the conventional test which was performed employing a fixed amount of PDP.

In order further to confirm the reliability of the MG-PDP test, a total of 688 Micrococcaceae strains (95 micrococci and 593 staphylococci) were identified and subsequently inoculated in M9 plates containing $200 \mu \mathrm{g} / \mathrm{ml}$ PDP to test phosphatase production according to the conventional technique, and in M9 plates containing $100 \mu \mathrm{g} / \mathrm{ml}$ PDP plus $25 \mu \mathrm{g} / \mathrm{ml} \mathrm{MG}$ to test their green staining. A complete correlation between deep-green staining on MGPDP medium and phosphatase activity of the strains was found (Table 1). These results appear to agree with most recent data in the literature concerning phosphatase production by the Micrococcaceae (Kloos and Schleifer, 1975; Subcomittee on the Taxonomy of Staphylococci and Micrococci, 1976; Oeding and Digranes, 1977; Varaldo and Satta, 1978).

As for micro-organisms other than the Micrococcaceae, the MG-PDP phosphatase test could also be performed directly on the media most commonly used for isolating streptococci, corynebacteria, and candidae (Table 2). An unclear and aspecific staining of phosphatase-positive colonies of enterobacteria was obtained using MacConkey-base medium. This could be due either to the presence of a $\mathrm{pH}$ indicating dye or to alterations in cell wall permeability by bile salts in the medium.

FACTORS AFFECTING MG-PDP PHOSPHATASE TEST

The relevance of different factors in the MG-PDP phosphatase test was analysed and is briefly summarised here.

The test is not grossly affected by the type of 
Table 1 Phosphatase activity of 688 strains of the Micrococcaceae as revealed by green colony staining on MG-PDP M9 medium

\begin{tabular}{lcll}
\hline Species & $\begin{array}{l}\text { No. of } \\
\text { strains }\end{array}$ & $\begin{array}{l}\text { Phosphatase } \\
\text { activity } \\
\text { (conventional MG-PDP } \\
\text { test) }\end{array}$ & $\begin{array}{l}\text { Green colony } \\
\text { stain on }\end{array}$ \\
\hline Micrococcus luteus & 56 & - & - \\
M. varians & 29 & - & - \\
M. roseus & 7 & - & - \\
M. lylae & 2 & - & - \\
M. sedentarius & 1 & - & - \\
Staphyloccus aureus & 251 & + & - \\
Staph. epidermidis & 132 & + & - \\
Staph. haemolyticus & 11 & - & - \\
Staph. hominis & 71 & - & - \\
Staph. saprophyticus & 62 & - & - \\
Staph. xylosus & 20 & - & - \\
Staph. simulans & 12 & + & +- \\
Staph. capitis & 3 & - & - \\
Staph. cohnii & 14 & - & - \\
Staph. warneri & 12 & - & - \\
\hline & 4 & - & - \\
\hline
\end{tabular}

base medium, unless the latter contains $\mathrm{pH}$ indicators or other substances causing heavy staining of bacterial colonies which could interfere with or mask the MG uptake (Table 2). The MG-PDP test works equally well on media ranging between $\mathrm{pH} 5.5$ and 8.0. It should only be mentioned that the MG con- centration in the medium is reducible at acidic $\mathrm{pH}$, as the lower the $\mathrm{pH}$ the more intense the stain. Plate storage does not alter the specific staining of phosphatase-positive colonies (a better differential stain is obtained with MG-PDP plates stored for four weeks at $4^{\circ} \mathrm{C}$ than with the same plates freshly prepared). As for salt concentration, the differential staining of phosphatase-positive and -negative colonies is sharper in M9 medium deprived of $\mathrm{NaCl}$ and is reduced in media containing $1.5 \%$ or more $\mathrm{NaCl}$.

\section{Discussion}

Out of 739 strains belonging to various taxonomic groups, all of the phosphatase-positive strains, but none of the phosphatase-negative strains, were found to produce deep-green-stained colonies when grown on MG-PDP media. In addition, phosphatasepositive colonies of candidae did not take up the green stain under phosphatase-inhibiting conditions, where the conventional phosphatase test also gave negative results. Finally, the phosphatase-positive micro-organisms grew unstained colonies unless a substrate for phosphatase such as PDP, besides MG, was included in the medium. These findings clearly

Table 2 Summary of various MG-PDP media and their potential use as detectors of phosphatase activity

\begin{tabular}{|c|c|c|c|c|}
\hline \multirow[t]{2}{*}{ Microorganisms } & \multirow[t]{2}{*}{ Base medium } & \multirow{2}{*}{$\begin{array}{l}\text { Concentration } \\
M G-P D P(\mu g / m l)\end{array}$} & \multicolumn{2}{|c|}{ Green colony staining } \\
\hline & & & $\begin{array}{l}\text { Phosphatase- } \\
\text { positive strains }\end{array}$ & $\begin{array}{l}\text { Phosphatase- } \\
\text { negative strains }\end{array}$ \\
\hline \multirow[t]{7}{*}{ Micrococcaceae } & M9 & $25-200$ & ++ & - \\
\hline & TPA & $25-200$ & $+t$ & - \\
\hline & BAB & $25-200$ & $+t$ & - \\
\hline & BHIA & $25-200$ & + & - \\
\hline & MH & $25-200$ & + & - \\
\hline & DST & $25-200$ & + & - \\
\hline & BP & $\mathrm{U}$ & - & - \\
\hline \multirow[t]{3}{*}{ Streptococci } & M9 & $50-200$ & ++ & - \\
\hline & TPA & $50-200$ & $+t$ & - \\
\hline & $\mathrm{TH}$ & $50-200$ & ++ & - \\
\hline \multirow[t]{4}{*}{ Corynebacteria } & M9 & $50-800$ & ++ & - \\
\hline & TPA & $50-800$ & $+t$ & - \\
\hline & LM & $50-1000$ & ++ & - \\
\hline & MT & $\mathbf{U}$ & - & - \\
\hline \multirow[t]{4}{*}{ Enterobacteria } & M9 & $500-800$ & $+t$ & - \\
\hline & TPA & $500-800$ & ++ & - \\
\hline & MC & $\mathbf{U}$ & - & - \\
\hline & DCC. & $\mathbf{U}$ & - & - \\
\hline \multirow[t]{6}{*}{ Candidae } & M9 (pH 5.5) & $25-400$ & $+t$ & - \\
\hline & SD & $25-400$ & ++ & - \\
\hline & CSA & $25-400$ & + & \pm \\
\hline & MA & $25-400$ & + & \pm \\
\hline & M9 & $\mathbf{U}$ & - & - \\
\hline & TPA & $\mathbf{U}$ & - & - \\
\hline
\end{tabular}

Abbreviations: M9, M9 medium (Satta et al., 1977); TPA, tryptose phosphate broth (Difco) supplemented with 1.5\% agar; BAB, blood agar base (Difco); BHIA, brain heart infusion agar (Difco); MH, Mueller Hinton medium (Difco); DST, DST agar (Oxoid); BP, Baird-Parker agar base (BBL) supplemented with EY-tellurite enrichment (Difco); TH, Todd-Hewitt broth (Oxoid) supplemented with $1 \cdot 5 \%$ agar; LM, Loeffler medium (BBL); MT Mueller tellurite base (Difco) enriched with Mueller tellurite serum (Difco); MC, MacConkey agar (Difco); DCC, desoxycholate citrate agar, Hynes modification (Oxoid); SD, Sabouraud dextrose medium (Difco); CSA, Czapek solution agar (Difco), pH 5.5; MA, Mycobiotic agar (Difco), pH 5.5.

$\mathbf{U}=$ undetermined. 
indicate that the relationships between phosphatase production and MG uptake by colonies growing on MG-PDP media cannot be purely circumstantial. The green colony stain, on the contrary, appears to be directly related to phosphatase activity.

An interesting characteristic of the MG-PDP phosphatase test is that phosphatase-positive colonies stain heavily against the agar background, with no zone of staining in the surrounding medium. Moreover, phosphatase-positive bacterial cells grown on MG-PDP media display a green staining under light microscopy. These findings indicate that phosphatase-positive cells, but not phosphatasenegative cells, are capable of transporting and concentrating the MG stain into the cytoplasm. Although this phenomenon is not yet understood, preliminary experiments allow the suggestion of likely explanations. Using several other stains, no intracytoplasmic stain concentration by phosphatase-positive cells is observed. Furthermore, when staphylococci are grown on plates containing $25 \mu \mathrm{g} / \mathrm{ml} \mathrm{MG}$ and $200 \mu \mathrm{g} / \mathrm{ml}$ PDP (the latter previously digested with bovine phosphatase) no differential staining between phosphatase-positive and -negative strains is observed. From these findings, it is unlikely that MG interacts either with phenolphthalein or with phosphate ions, after phosphatase reaction, yielding a product which is then actively transported into the cells. Rather, active concentration of MG appears to be dependent on a phosphatase reaction occurring in the cells. This reaction could activate both a transport system specific for phosphate ions and transport system(s) for another substance(s) such as MG. It is also possible that, in the bacterial cells tested, the phosphate transport system is shared by MG, as the capability of transporting different substances by a unique transport system is well established in bacteria (Oxender, 1972). Alternatively, the splitting of the phosphate bonds of a substrate, such as PDP, by phosphatase could liberate energy for the active concentration of MG inside cells.

From a practical point of view, the MG-PDP phosphatase test appears to be at least as sensitive and specific as the conventional phosphatase tests (see also Oeding and Digranes, 1977). The advantages of the MG-PDP test as compared to other methods are evident. Firstly, after the appropriate medium inoculation, no additional step is needed to read the test. This particular feature appears to be of great importance because it allows the checking of the phosphatase activity of the colonies at any given time without the risk of contaminating or killing the strains under study; this is particularly advantageous in the case of weak phosphataseproducing or slowly growing strains. Furthermore, the test can be performed using many of the selective media that are employed for primary isolation of various micro-organisms. Secondly, the MG-PDP test allows an easy evaluation of phosphatase activity also of colonies grown in deep agar. Such a property makes this test particularly suitable for studying phosphatase production among anaerobic bacteria. Thirdly, multitest kits and micromethods are being extensively employed for the rapid identification of both aerobic and anaerobic bacteria, and in this regard a phosphatase test resulting in a reliable staining of phosphatase-positive colonies would certainly be preferred to conventional techniques. Finally, by the MG-PDP test phosphatase-positive and -negative colonies can be distinguished even in crowded-growth conditions; no other phosphatase test so far described can offer this particular advantage, which cannot only prove useful in routine clinical bacteriology but may also be of great importance for genetic and physiological studies of bacterial phosphatases.

\section{References}

Baird-Parker, A. C. (1963). A classification of micrococci and staphylococci based on physiological and biochemical tests. Journal of General Microbiology, 30, 409-427.

Barber, M., and Kuper, S. W. A. (1951). Identification of Staphylococcus pyogenes by the phosphatase reaction. Journal of Pathology and Bacteriology, 63, 65-68.

Bayliss, M., Glick, D., and Siem, R. A. (1948). Demonstration of phosphatases and lipase in bacteria and true fungi by staining methods and the effect of penicillin on phosphatase activity. Journal of Bacteriology, 55, 307-316.

Bhatti, A. R., and Done, J. (1974). On the presence of two types of alkaline phosphatase in Serratia marcescens. Microbios, 11, 203-213.

Bray, J., and King, E. J. (1943). The phosphatase reaction as an aid to the identification of microorganisms using phenolphthalein phosphate as substrate. Journal of Pathology and Bacteriology, 55, 315-320.

King, E. J. (1943). Preparation of phenolphthalein phosphate. Journal of Pathology and Bacteriology, 55, 311-314.

Kloos, W. E., and Schleifer, K. H. (1975). Isolation and characterization of staphylococci from human skin. II. Descriptions of four new species: Staphylococcus warneri, Staphylococcus capitis, Staphylococcus hominis, and Staphylococcus simulans. International Journal of Systematic Bacteriology, 25, 62-79.

Kloos, W. E., Tornabene, T. G., and Schleifer, K. H. (1974). Isolation and characterization of micrococci from human skin, including two new species: Micrococcus lylae and Micrococcus kristinae. International Journal of Systematic Bacteriology, 24, 79-101.

Lennette, E. H., Spaulding, E. G., and Truant, J. P. 
(1974). Manual of Clinical Microbiology, 2nd edition. American Society for Microbiology, Washington, DC.

Oeding, P., and Digranes, A. (1977). Classification of coagulase-negative staphylococci in the diagnostic laboratory. Acta Pathologica et Microbiologica Scandinavica, 85B, 136-142.

Oxender, D. L. (1972). Amino acid transport in microorganisms. In Metabolic Pathways, Vol. 5, 'Metabolic Transport', edited by L. E. Hokin, pp. 133-185. Academic Press, New York.

Porschen, R. K., and Spaulding, E. H. (1974). Phosphatase activity of anaerobic organisms. Applied Microbiology, 27, 744-747.

Satta, G., Varaldo, P. E., Grazi, G., and Fontana, R. (1977). Bacteriolytic activity in staphylococci. Infection and Immunity, 16, 37-42.

Schleifer, K. H., and Kloos, W. E. (1975). Isolation and characterization of staphylococci from human skin. I. Amended descriptions of Staphylococcus epidermidis and Staphylococcus saprophyticus, and descriptions of three new species: Staphylococcus cohnii, Staphylococcus haemolyticus and Staphylococcus xylosus. International Journal of Systematic Bacteriology, 25, 50-61.

Smith, R. F., Blasi, D., and Dayton, S. L. (1973).
Phosphatase activity among Candida species and other yeasts isolated from clinical material. Applied Microbiology, 26, 364-367.

Subcommittee on the Taxonomy of Staphylococci and Micrococci (1976). Minutes of the Meeting, 9 September 1975. Appendix 1, Identification of Staphylococci. International Journal of Systematic Bacteriology, 26, 332-334.

Taketo, Y., and Taketo, A. (1974). Phosphoesterases of hemolytic streptococci. Japanese Journal of Experimental Medicine, 44, 291-299.

Varaldo, P. E., and Satta, G. (1978). Grouping of staphylococci on the basis of their bacteriolyticactivity patterns: a new approach to the taxonomy of the Micrococcaceae. II, Main characters of the 1054 strains subdivided into 'lyogroups'. International Journal of Systematic Bacteriology, 28, 148-153.

Wolf, P. L., Von Der Muehll, E., and Ludwick, M. (1972). A new test to differentiate Serratia from Enterobacter. American Journal of Clinical Pathology, 57, 241-245.

Requests for reprints to: Dr Giuseppe Satta, Istituto di Microbiologia dell'Università di Genova, Viale Benedetto XV, 10, 16132 Genova, Italy 